\title{
A 2DOF hybrid energy harvester based on combined piezoelectric and electromagnetic conversion mechanisms"
}

\author{
Hong-yan WANG ${ }^{\dagger 1,2}$, Li-hua TANG ${ }^{3}$, Yuan GUO ${ }^{1}$, Xiao-biao SHAN $^{2}$, Tao XIE ${ }^{2}$ \\ $\left({ }^{1}\right.$ College of Computer and Control Engineering, Qiqihar University, Qiqihar 161006, China) \\ ( ${ }^{2}$ State Key Laboratory of Robotics and System, Harbin Institute of Technology, Harbin 150001, China) \\ ( ${ }^{3}$ Department of Mechanical Engineering, University of Auckland, Auckland 1010, New Zealand) \\ †E-mail: wanghongyan1993@163.com \\ Received May 4, 2014; Revision accepted July 24, 2014; Crosschecked Aug. 25, 2014
}

\begin{abstract}
This paper presents a two-degree-of-freedom (2DOF) hybrid piezoelectric-electromagnetic energy harvester (P-EMEH). Such a 2DOF system is designed to achieve two close resonant frequencies. The combined piezoelectricelectromagnetic conversion mechanism is exploited to further improve the total power output of the system in comparison to a stand-alone piezoelectric or electromagnetic conversion mechanism. First, a mathematical model for the 2DOF hybrid P-EMEH is established. Subsequently, the maximal power output of the 2DOF hybrid P-EMEH is compared both experimentally and theoretically with those from the 1DOF piezoelectric energy harvester (PEH), 1DOF electromagnetic energy harvester (EMEH), 2DOF $\mathrm{PEH}$, and 2DOF EMEH. Based on the validated mathematical model, the effect of the effective electromechanical coupling coefficients (EMCC) on the maximal power outputs from various harvester configurations is analyzed. The results indicate that for the 2DOF hybrid P-EMEH, although the increase of the power output from one electromechanical transducer will lead to the decrease of the power output from the other, the overall performance of the system is improved in weak and medium coupling regimes by increasing electromechanical coupling. In weak and medium coupling scenarios, the hybrid energy harvester configuration is advantageous over conventional $1 \mathrm{DOF}$ or $2 \mathrm{DOF}$ harvester configurations with a stand-alone conversion mechanism.
\end{abstract}

Key words: Vibration, Two-degree-of-freedom (2DOF), Hybrid piezoelectric-electromagnetic conversion, Energy harvesting doi: 10.1631 jzus.A1400124

Document code: A

CLC number: TM619; TN384

\section{Introduction}

Over the past few years, vibration energy harvesting has attracted much research interest owing to its potential to implement low-cost self-powered wireless sensors. Some methods are commonly pursued to harness electrical energy from ambient vibrations, including electrostatic generation (Lallart et al., 2011), electromagnetic induction (Elvin and Elvin, 2011; Jung et al., 2011), and the piezoelectric effect

\footnotetext{
* Project supported by the National Natural Science Foundation of China (No. 51077018), the Heilongjiang Provincial Natural Science Fundation (No. F201219), and the Program for Young Teachers Scientific Research in Qiqihar University (No. 2012k-Z12), China (C) Zhejiang University and Springer-Verlag Berlin Heidelberg 2014
}

(Yang et al., 2009; Lumentut and Howard, 2011; Wang et al., 2012; 2014). No matter which method is adopted, the efficiency improvement of a vibration energy harvester involves effort in terms of both bandwidth enlargement and magnitude amplification.

Conventional vibration energy harvesters are usually designed as one-degree-of-freedom (1DOF) models (Williams and Yates, 1996; Roundy et al., 2003; Dutoit et al., 2005), which are efficient near a single resonant frequency. Narrow operating bandwidth is a major challenge for $1 \mathrm{DOF}$ vibration energy harvesters (Tang et al., 2013). To overcome that drawback, some researchers have considered the use of two-degree-of-freedom (2DOF) models to achieve two close resonances for wider bandwidth. Aldraihem and Baz (2011) and Arafa et al. (2011) proposed a 
2DOF piezoelectric energy harvester $(\mathrm{PEH})$ model comprising a piezoelectric harvester and an additional spring-mass system installed between the harvester and the base. The additional spring-mass system served as a dynamic magnifier to enhance the power output and meanwhile achieve a wider bandwidth. Tang and Yang (2012) presented a 2DOF PEH model comprising dual spring-mass-dampers connected in series, where a piezoelectric transducer for power generation was placed between the primary mass and the base, and a parasitic mass connected to the primary mass was used to achieve two close resonant frequencies. Harne (2012) established the mathematical models of a 2DOF electromagnetic energy harvester (EMEH) and a 2DOF PEH, respectively. With these established models, a parametric study was performed to investigate the energy harvesting efficiency of these two types of 2DOF harvesters.

On the other hand, most studies in the energy harvesting field have focused on stand-alone conversion mechanisms (electrostatic, electromagnetic, or piezoelectric). Piezoelectric and electrostatic mechanisms are suitable for small size harvesters in view of their compatibility with standard microelectromechnical systems (MEMS) manufacturing procedure, while electromagnetic mechanisms are more suitable for large size harvesters. In recent studies, the hybrid energy harvesting technology is being increasingly investigated (Khaligh et al., 2008; Wacharasindhu and Kwon, 2008; Challa et al., 2009; Tadesse et al., 2009; Yang et al., 2010; Kim et al., 2012; Shan et al., 2013). Usually, for hybrid energy harvesting, the PEH and EMEH should be on the same scale (for comparable power output), otherwise the hybrid scheme will be meaningless. Tadesse et al. (2009) proposed a multimodal energy harvesting device that combines piezoelectric and electromagnetic energy harvesting mechanisms. They found that the electromagnetic transducer could generate much higher power at lower frequencies (the first transversal resonance mode), while the piezoelectric transducer does so at higher frequencies (the second transversal resonance mode). Thus, the combination of the two transducers into a device can generate higher power outputs covering multiple modes. Challa et al. (2009) presented a coupled piezoelectric-electromagnetic energy harvesting technique, where two independent energy harvesting mechanisms are coupled to provide higher electrical damping so as to match it better to the mechanical damping in the system for an increased power output. However, questions, such as how the two conversion mechanisms affect each other and how the coupling strengths affect overall performance after combination, have not been properly answered, especially for a multiple DOF energy harvesting system.

This paper proposes a 2DOF hybrid piezoelectricelectromagnetic energy harvester (P-EMEH) system, which aims at not only achieving wider bandwidth but also an increased output by combining piezoelectric and electromagnetic components of comparable macroscopic size. A mathematical model for the 2DOF hybrid P-EMEH system is established. The system parameters for model validation are determined from experiment. The maximal power output from the 2DOF hybrid P-EMEH is compared both experimentally and theoretically with those from other energy harvesting configurations, including 1DOF PEH, 2DOF PEH, 1DOF EMEH, and 2DOF EMEH. The advantage of using the 2DOF hybrid P-EMEH is discussed. Finally, the effect of the effective electromechanical coupling coefficients (EMCC) on the maximal power outputs from various harvester configurations is investigated.

\section{Proposed model of 2DOF hybrid P-EMEH}

A conventional vibration energy harvester is designed as a 1DOF lumped parameter model. Figs. 1a and $1 \mathrm{~b}$ show a 1DOF PEH model and a 1DOF EMEH model, respectively. The $1 \mathrm{DOF}$ PEH model comprises a mass $M_{1}$, spring $K_{1}$, damping $C_{1}$, and the piezoelectric element. The piezoelectric element is placed between the base and the mass, generating alternating electrical output to power the resistor $R_{\mathrm{L}}$. The 1DOF EMEH model comprises the mass $M_{2}$, spring $K_{2}$, and damper $C_{2}$, where the magnetic mass $M_{2}$ vibrates through the axis of a wound coil. The coil is moving together with the base motions and the current is induced in the electromagnetic energy harvesting coil, delivering power to the resistor $R_{\mathrm{L} 2}$.

The 1DOF energy harvester model is only efficient near its sole resonant frequency. This drawback of the 1DOF model limits its applicability in the majority of practical vibration scenarios. In recent studies, some researchers have proposed 2DOF 
energy harvester models to achieve two closer resonant frequencies. Figs. 1c and 1d show a 2DOF PEH model (Tang and Yang, 2012) and a 2DOF EMEH model, respectively. In Figs. 1c and 1d, a 2DOF PEH model and a 2DOF EMEH model have the same mechanical subsystems and different electrical subsystems for power generation. For the mechanical subsystem of these two models, a mass $M_{1}$ with spring $K_{1}$ and damping $C_{1}$ is connected to the base, and the mass $M_{2}$ with spring $K_{2}$ and damping $C_{2}$ is connected to the mass $M_{1}$. For the electrical subsystem of the 2DOF PEH model, a piezoelectric transducer for voltage generation is placed between the mass $M_{1}$ and the base (Fig. 1c). For the electrical subsystem of the 2DOF EMEH model, a coil for the current generation is placed in the axis of the motion of the magnet mass $M_{2}$ (Fig. 1d).

The mass $M_{2}$ of the 2DOF PEH model (Fig. 1c) or the mass $M_{1}$ of the 2DOF EMEH model (Fig. 1d) is only used to achieve two close resonant frequencies and thus a wider operating bandwidth compared to 1DOF harvester. Actually, they can be further exploited to convert mechanical energy into electrical energy using an additional transducer. We propose a 2DOF hybrid P-EMEH model (Fig. 1e). As the base vibrates, the piezoelectric transducer placed between the base and the mass $M_{1}$ is deformed to generate the voltage across the resistor $R_{\mathrm{L} 1}$ by the piezoelectric effect. At the same time, the magnetic mass $M_{2}$ moves in and out of the coil and the coil generates induced current flowing through the resistor $R_{\mathrm{L} 2}$ according to Faraday's law. In Fig. $1, u_{0}, u_{1}$, and $u_{2}$ are the absolute coordinates of the base, $M_{1}$, and $M_{2}$, respectively. With this design, we aim at exploiting the full potential of the additional mechanical subsystem in a conventional 2DOF energy harvester model.

\section{Analytical modelling of 2DOF hybrid P-EMEH}

In Fig. 1e, setting $r_{1}=u_{1}-u_{0}$ and $r_{2}=u_{2}-u_{1}$, the mechanical governing equation of the 2DOF hybrid P-EMEH system can be written as

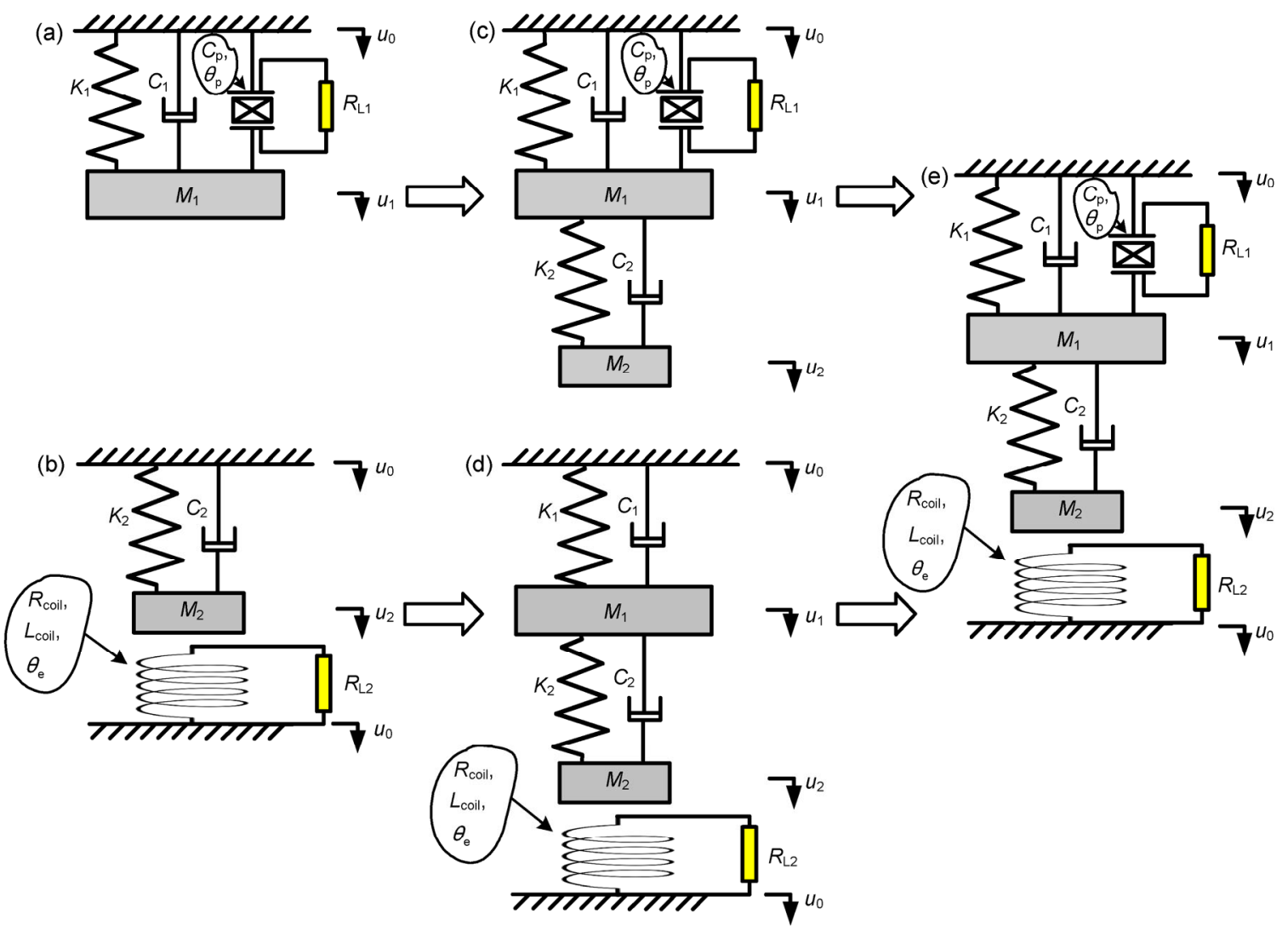

Fig. 1 Various energy harvester models: (a) 1DOF PEH; (b) 1DOF EMEH; (c) 2DOF PEH (Tang and Yang, 2012); (d) 2DOF EMEH; (e) 2DOF hybrid P-EMEH 


$$
\left\{\begin{array}{c}
\left(M_{1}+M_{2}\right) \ddot{r}_{1}+C_{1} \dot{r}_{1}+K_{1} r_{1}+M_{2} \ddot{r}_{2}+F_{\mathrm{p}}+F_{\mathrm{e}} \\
=-\left(M_{1}+M_{2}\right) \ddot{u}_{0}, \\
M_{2} \ddot{r}_{2}+C_{2} \dot{r}_{2}+K_{2} r_{2}+F_{\mathrm{e}}=-M_{2} \ddot{r}_{1}-M_{2} \ddot{u}_{0},
\end{array}\right.
$$

where $K_{1}$ and $K_{2}$ are the spring stiffness of mass $M_{1}$ and $M_{2}$, respectively. $C_{1}$ and $C_{2}$ are the mechanical damping of mass $M_{1}$ and $M_{2}$, respectively. As the 2DOF hybrid P-EMEH delivers energy to the load resistances, the mechanical domain receives the feedback from the electrical domain, which is represented by the backward electromechanical coupling forces, $F_{\mathrm{p}}$ and $F_{\mathrm{e}} . F_{\mathrm{p}}$ is the backward coupling force due to the creation of the voltage across the piezoelectric element, which is given by

$$
F_{\mathrm{p}}=\theta_{\mathrm{p}} V_{\mathrm{R} 1},
$$

where $V_{\mathrm{R} 1}$ is the voltage across the resistor $R_{\mathrm{L} 1}$, and $\theta_{\mathrm{p}}$ is the piezoelectric coupling coefficient. $F_{\mathrm{e}}$ is the backward coupling force due to the creation of an opposing magnetic field by the current flowing through the coil, which is given by

$$
F_{\mathrm{e}}=\theta_{\mathrm{e}} I_{\mathrm{R} 2}
$$

where $I_{\mathrm{R} 2}$ is the current flowing through the resistor $R_{\mathrm{L} 2}$, and $\theta_{\mathrm{e}}$ is the electromagnetic coupling coefficient, $\theta_{\mathrm{e}}=B_{1} l$, where $B_{1}$ and $l$ are the average magnetic flux density and the total length of the coil, respectively.

The simplified electrical models of the piezoelectric and electromagnetic transducers are given in Fig. 2a and Fig. 2b, respectively. In Fig. 2a, the electrical model of the piezoelectric transducer consists of a current source $I_{\mathrm{p}}$ in parallel with its internal capacitance $C_{\mathrm{p}}$, where the output terminal is connected with the resistor $R_{\mathrm{L} 1}$. In Fig. 2b, the electrical model of the electromagnetic transducer consists of a voltage source $V_{\mathrm{e}}$ in series with its internal inductance $L_{\text {coil }}$ and the coil resistance $R_{\text {coil }}$, where the output terminal is connected with the resistor $R_{\mathrm{L} 2}$.

According to Kirchhoff's current law, the circuitgoverning equation for piezoelectric transduction of the system (Fig. 2a) is written as

$$
-I_{\mathrm{p}}+C_{\mathrm{p}} \dot{V}_{\mathrm{R} 1}+\frac{V_{\mathrm{R} 1}}{R_{\mathrm{L} 1}}=0
$$

where the current $I_{\mathrm{p}}$ is proportional to the velocity $\dot{r}_{1}$ in the mechanical domain, i.e., $I_{\mathrm{p}}=\theta_{\mathrm{p}} \dot{r}_{1}$.

(a)

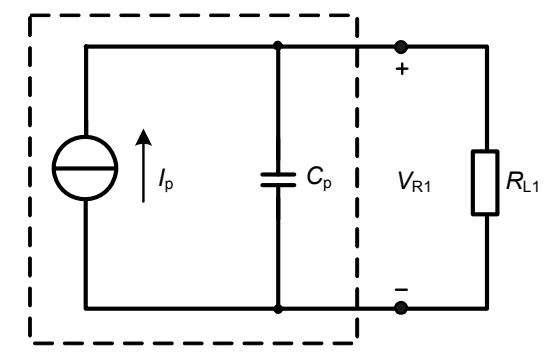

(b)

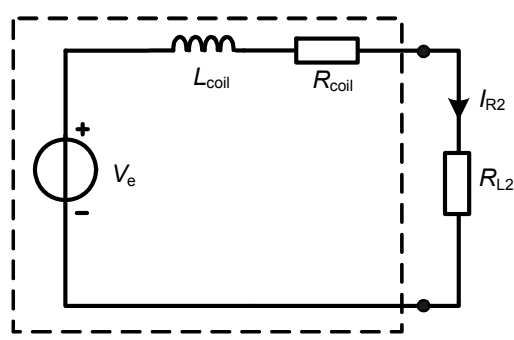

Fig. 2 Equivalent electrical models of piezoelectric transducer (a) and electromagnetic transducer (b)

According to Kirchhoff's voltage law, the circuitgoverning equation for electromagnetic transduction of the system (Fig. 2b) is written as

$$
-V_{\mathrm{e}}+L_{\text {coil }} \dot{I}_{\mathrm{R} 2}+\left(R_{\text {coil }}+R_{\mathrm{L} 2}\right) I_{\mathrm{R} 2}=0
$$

where the voltage $V_{\mathrm{e}}$ is proportional to the velocity $\left(\dot{r}_{1}+\dot{r}_{2}\right)$ in the mechanical domain, i.e., $V_{\mathrm{e}}=\theta_{\mathrm{e}}\left(\dot{r}_{1}+\dot{r}_{2}\right)$.

Substituting Eqs. (2) and (3) into Eq. (1) and further combining Eqs. (1), (4), and (5), the electromechanical model of the 2DOF hybrid P-EMEH is written as

$$
\left\{\begin{array}{l}
\left(M_{1}+M_{2}\right) \ddot{r}_{1}+C_{1} \dot{r}_{1}+K_{1} r_{1}+M_{2} \ddot{r}_{2}+\theta_{\mathrm{p}} V_{\mathrm{R} 1}+\theta_{\mathrm{e}} I_{\mathrm{R} 2} \\
=-\left(M_{1}+M_{2}\right) \ddot{u}_{0}, \\
M_{2} \ddot{r}_{2}+C_{2} \dot{r}_{2}+K_{2} r_{2}+\theta_{\mathrm{e}} I_{\mathrm{R} 2}=-M_{2} \ddot{r}_{1}-M_{2} \ddot{u}_{0}, \\
-\theta_{\mathrm{p} 1} \dot{r}_{1}+C_{\mathrm{p}} \dot{V}_{\mathrm{R} 1}+V_{\mathrm{R} 1} / R_{\mathrm{L} 1}=0, \\
-\theta_{\mathrm{e}}\left(\dot{r}_{1}+\dot{r}_{2}\right)+L_{\text {coil }} \dot{I}_{\mathrm{R} 2}+\left(R_{\mathrm{coil}}+R_{\mathrm{L} 2}\right) I_{\mathrm{R} 2}=0 .
\end{array}\right.
$$

Letting $\omega_{1}=\sqrt{K_{1} / M_{1}}, \omega_{2}=\sqrt{K_{2} / M_{2}}, \zeta_{1}=C_{1} /\left(2 M_{1} \omega_{1}\right)$, $\zeta_{2}=C_{2} /\left(2 M_{2} \omega_{2}\right)$, and $u=M_{2} / M_{1}$, and applying the Laplace transform for Eq. (6), we can obtain: 


$$
\left\{\begin{array}{c}
{\left[(1+u) s^{2}+2 \zeta_{1} \omega_{1} s+\omega_{1}^{2}\right] \hat{r}_{1}+u s^{2} \hat{r}_{2}+\left(\theta_{\mathrm{p}} / M_{1}\right) V_{\mathrm{R} 1}} \\
\quad+\left(\theta_{\mathrm{e}} / M_{1}\right) \hat{I}_{\mathrm{R} 2}=-(1+u) s^{2} \hat{u}_{0} \\
\left(s^{2}+2 \zeta_{2} \omega_{2} s+\omega_{2}^{2}\right) \hat{r}_{2}+\left(\frac{\theta_{\mathrm{e}}}{u M_{1}}\right) \hat{I}_{\mathrm{R} 2}=-s^{2} \hat{r}_{1}-s^{2} \hat{u}_{0} \\
-\theta_{\mathrm{p}} s \hat{r}_{1}+C_{\mathrm{p}} s \hat{V}_{\mathrm{R} 1}+\hat{V}_{\mathrm{R} 1} / R_{\mathrm{L} 1}=0 \\
-\theta_{\mathrm{e}} s\left(\hat{r}_{1}+\hat{r}_{2}\right)+L_{\mathrm{coi}} s \hat{I}_{\mathrm{R} 2}+\left(R_{\mathrm{coil}}+R_{\mathrm{L} 2}\right) \hat{I}_{\mathrm{R} 2}=0
\end{array}\right.
$$

where $s$ is the Laplace variable, $\omega_{1}$ and $\omega_{2}$ are the natural angular frequencies when the two subsystems work separately.

Solving Eq. (7) and letting $s=\mathrm{j} \omega$, the voltage across the resistor $R_{\mathrm{L} 1}$ is obtained as

$$
\begin{aligned}
& \left|\frac{\hat{V}_{\mathrm{R} 1}}{\omega^{2} \hat{u}_{0}}\right|=\frac{M_{1}}{\left|\theta_{\mathrm{p}}\right|} \mid\left(1+\frac{u\left(\alpha^{2}+\mathrm{j} 2 \zeta_{2} \alpha \Omega_{1}\right)}{A}\right) \\
& /\left(\left(1-\Omega_{1}^{2}+\mathrm{j} 2 \zeta_{1} \Omega_{1}+u\left(\alpha^{2}+\mathrm{j} 2 \zeta_{2} \alpha \Omega_{1}\right)\right.\right. \\
& \left.\left.-u\left(\alpha^{2}+\mathrm{j} 2 \zeta_{2} \alpha \Omega_{1}\right)^{2} / A\right) \frac{\mathrm{j} \omega_{\mathrm{p}} \Omega_{1}+1}{\mathrm{j} k_{\mathrm{p}}^{2} \omega_{\mathrm{p}} \Omega_{1}}+1\right),
\end{aligned}
$$

and the current through the resistor $R_{\mathrm{L} 2}$ is obtained as

$$
\begin{aligned}
& \left|\frac{\hat{I}_{\mathrm{R} 2}}{\omega^{2} \hat{u}_{0}}\right|=\frac{u M_{1}}{\left|\theta_{\mathrm{e}}\right|} \mid\left(1+\frac{\alpha^{2}+\mathrm{j} 2 \zeta_{2} \alpha \Omega_{1}}{B}\right) \\
& \quad /\left(\left(\alpha^{2}-\Omega_{1}^{2}+\mathrm{j} 2 \zeta_{2} \alpha \Omega_{1}-u\left(\alpha^{2}+\mathrm{j} 2 \zeta_{2} \alpha \Omega_{1}\right)^{2} / B\right)\right. \\
& \left.\quad \times \frac{\mathrm{j} \Omega_{1}+\alpha \omega_{\mathrm{e}}}{\mathrm{j} k_{\mathrm{e}}^{2} \alpha^{2} \Omega_{1}}+1\right) \mid
\end{aligned}
$$

where $\mathrm{j}$ is the imaginary unit, $\omega$ is the excitation angular frequency, and the dimensionless parameters are

$$
\begin{aligned}
& \alpha=\frac{\omega_{2}}{\omega_{1}}, \Omega_{1}=\frac{\omega}{\omega_{1}}, k_{\mathrm{p}}^{2}=\frac{\theta_{\mathrm{p}}^{2}}{C_{\mathrm{p}} K_{1}}, \omega_{\mathrm{p}}=R_{\mathrm{L} 1} C_{\mathrm{p}} \omega_{1}, \\
& k_{\mathrm{e}}^{2}=\frac{\theta_{\mathrm{e}}^{2}}{L_{\text {coil }} K_{2}}, \omega_{\mathrm{e}}=\frac{R_{\text {coil }}+R_{\mathrm{L} 2}}{\omega_{2} L_{\text {coil }}}, \\
& A=\alpha^{2}-\Omega_{1}^{2}+\mathrm{j} 2 \zeta_{2} \alpha \Omega_{1}+\frac{\mathrm{j} k_{\mathrm{e}}^{2} \alpha^{2} \Omega_{1}}{\mathrm{j} \Omega_{1}+\alpha \omega_{\mathrm{e}}}, \\
& B=1-\Omega_{1}^{2}+\mathrm{j} 2 \zeta_{1} \Omega_{1}+u\left(\alpha^{2}+\mathrm{j} 2 \zeta_{2} \alpha \Omega_{1}\right)+\frac{\mathrm{j} k_{\mathrm{p}}^{2} \omega_{\mathrm{p}} \Omega_{1}}{\mathrm{j} \omega_{\mathrm{p}} \Omega_{1}+1} .
\end{aligned}
$$

The power outputs on the resistors $R_{\mathrm{L} 1}$ and $R_{\mathrm{L} 2}$ are then obtained as

$$
\begin{gathered}
\left|\frac{\hat{P}_{\mathrm{R} 1}}{\left(\omega^{2} \hat{u}_{0}\right)^{2}}\right|=\frac{M_{1}}{\omega_{1} \omega_{\mathrm{p}} k_{\mathrm{p}}^{2}} \mid\left(1+\frac{u\left(\alpha^{2}+\mathrm{j} 2 \zeta_{2} \alpha \Omega_{1}\right)}{A}\right) \\
/\left(\left(1-\Omega_{1}^{2}+\mathrm{j} 2 \zeta_{1} \Omega_{1}+u\left(\alpha^{2}+\mathrm{j} 2 \zeta_{2} \alpha \Omega_{1}\right)\right.\right. \\
\left.\left.-u\left(\alpha^{2}+\mathrm{j} 2 \zeta_{2} \alpha \Omega_{1}\right)^{2} / A\right) \frac{\mathrm{j} \omega_{\mathrm{p}} \Omega_{1}+1}{\mathrm{j} k_{\mathrm{p}}^{2} \omega_{\mathrm{p}} \Omega_{1}}+1\right)\left.\right|^{2}, \\
\quad\left|\frac{\hat{P}_{\mathrm{R} 2}}{\left(\omega^{2} \hat{u}_{0}\right)^{2}}\right|=\frac{u M_{1} \omega_{\mathrm{e}}}{\alpha \omega_{1} k_{\mathrm{e}}^{2}\left(1+R_{\mathrm{coil}} / R_{\mathrm{L} 2}\right)} \\
\quad \mid\left(1+\frac{\alpha^{2}+\mathrm{j} 2 \zeta_{2} \alpha \Omega_{1}}{B}\right) /\left(\left(\alpha^{2}-\Omega_{1}^{2}+\mathrm{j} 2 \zeta_{2} \alpha \Omega_{1}\right.\right. \\
\left.\left.-u\left(\alpha^{2}+\mathrm{j} 2 \zeta_{2} \alpha \Omega_{1}\right)^{2} / B\right) \frac{\mathrm{j} \Omega_{1}+\alpha \omega_{\mathrm{e}}}{\mathrm{j} k_{\mathrm{e}}^{2} \alpha^{2} \Omega_{1}}+1\right)\left.\right|^{2} .
\end{gathered}
$$

The total power of the 2DOF hybrid P-EMEH energy harvester is

$$
\hat{P}_{\text {total }}=\hat{P}_{\mathrm{R} 1}+\hat{P}_{\mathrm{R} 2}
$$

As $R_{\mathrm{L} 2} \rightarrow \infty, \omega_{\mathrm{e}} \rightarrow \infty$, and $A \rightarrow \alpha^{2}-\Omega_{1}^{2}+\mathrm{j} 2 \zeta_{2} \alpha \Omega_{1}$, Eq. (12) is reduced to $\hat{P}_{\text {total }} \rightarrow \hat{P}_{\mathrm{R} 1}$, and Eq. (10) is reduced to

$$
\begin{gathered}
\left|\frac{\hat{P}_{\mathrm{R} 1}}{\left(\omega^{2} \hat{u}_{0}\right)^{2}}\right| \rightarrow \frac{M_{1}}{\omega_{1} \omega_{\mathrm{p}} k_{\mathrm{p}}^{2}} \mid\left(1+u+\frac{u \Omega_{1}^{2}}{\alpha^{2}-\Omega_{1}^{2}+\mathrm{j} 2 \zeta_{2} \alpha \Omega_{1}}\right) \\
/\left[\left(1-(1+u) \Omega_{1}^{2}+\mathrm{j} 2 \zeta_{1} \Omega_{1}\right.\right. \\
\left.\left.-\frac{u \Omega_{1}^{4}}{\alpha^{2}-\Omega_{1}^{2}+\mathrm{j} 2 \zeta_{2} \alpha \Omega_{1}}\right) \frac{\mathrm{j} \omega_{\mathrm{p}} \Omega_{1}+1}{\mathrm{j} k_{\mathrm{p}}^{2} \omega_{\mathrm{p}} \Omega_{1}}+1\right]\left.\right|^{2},
\end{gathered}
$$

which is the power output of the conventional 2DOF PEH (Fig. 1c). Furthermore, as $M_{2} \rightarrow 0$, the mass ratio $u \rightarrow 0$ and Eq. (13) is reduced to

$$
\begin{aligned}
& \left|\frac{\hat{P}_{\mathrm{R} 1}}{\left(\omega^{2} \hat{u}_{0}\right)^{2}}\right| \rightarrow \frac{M_{1}}{\omega_{1} \omega_{\mathrm{p}} k_{\mathrm{p}}^{2}} \\
& \quad \times\left|1 /\left(\left(1-\Omega_{1}^{2}+\mathrm{j} 2 \zeta_{1} \Omega_{1}\right) \frac{\mathrm{j} \omega_{\mathrm{p}} \Omega_{1}+1}{\mathrm{j} k_{\mathrm{p}}^{2} \omega_{\mathrm{p}} \Omega_{1}}+1\right)\right|^{2},
\end{aligned}
$$


which is the power output of the conventional $1 \mathrm{DOF}$ PEH (Fig. 1a).

As $R_{\mathrm{L} 1} \rightarrow 0, \omega_{\mathrm{p}} \rightarrow 0$, and $B \rightarrow 1-\Omega_{1}{ }^{2}+\mathrm{j} 2 \zeta_{1} \Omega_{1}+u\left(\alpha^{2}+\right.$ $\mathrm{j} 2 \zeta_{2} \alpha \Omega_{1}$ ), Eq. (12) is reduced to $\hat{P}_{\text {total }} \rightarrow \hat{P}_{\mathrm{R} 2}$, and Eq. (11) is reduced to

$$
\begin{aligned}
& \left|\frac{\hat{P}_{\mathrm{R} 2}}{\left(\omega^{2} \hat{u}_{0}\right)^{2}}\right| \rightarrow \frac{u M_{1} \omega_{\mathrm{e}}}{\alpha \omega_{1} k_{\mathrm{e}}^{2}\left(1+R_{\text {coil }} / R_{\mathrm{L} 2}\right)} \\
& \times \mid\left(1+\frac{\alpha^{2}+\mathrm{j} 2 \zeta_{2} \alpha \Omega_{1}}{1-\Omega_{1}^{2}+\mathrm{j} 2 \zeta_{1} \Omega_{1}+u\left(\alpha^{2}+\mathrm{j} 2 \zeta_{2} \alpha \Omega_{1}\right)}\right) \\
& \quad /\left[\left(\alpha^{2}-\Omega_{1}^{2}+\mathrm{j} 2 \zeta_{2} \alpha \Omega_{1}-u\left(\alpha^{2}+\mathrm{j} 2 \zeta_{2} \alpha \Omega_{1}\right)^{2}\right.\right. \\
& \left.\quad /\left(1-\Omega_{1}^{2}+\mathrm{j} 2 \zeta_{1} \Omega_{1}+u\left(\alpha^{2}+\mathrm{j} 2 \zeta_{2} \alpha \Omega_{1}\right)\right)\right) \\
& \left.\times \frac{\mathrm{j} \Omega_{1}+\alpha \omega_{\mathrm{e}}}{\mathrm{j} k_{\mathrm{e}}^{2} \alpha^{2} \Omega_{1}}+1\right]\left.\right|^{2},
\end{aligned}
$$

which is the power output of the conventional 2DOF EMEH (Fig. 1d).

In Eq. (15), letting $\Omega_{1}=\alpha \Omega_{2}, M_{1}=M_{2} / u$ and $\omega_{1}=\omega_{2} / \alpha$. As $K_{1} \rightarrow \infty$, the frequency ratio $\alpha \rightarrow 0$ and Eq. (15) is reduced to

$$
\begin{aligned}
& \left|\frac{\hat{P}_{\mathrm{R} 2}}{\left(\omega^{2} \hat{u}_{0}\right)^{2}}\right| \rightarrow \frac{M_{2} \Omega_{2}^{2} k_{\mathrm{e}}^{2} \omega_{\mathrm{e}}}{\omega_{2}\left(1+R_{\text {coil }} / R_{\mathrm{L} 2}\right)} \\
& \quad \times\left|\frac{1}{\left(1-\Omega_{2}^{2}+\mathrm{j} 2 \zeta_{2} \Omega_{2}\right)\left(\mathrm{j} \Omega_{2}+\omega_{\mathrm{e}}\right)+\mathrm{j} k_{\mathrm{e}}^{2} \Omega_{2}}\right|^{2},
\end{aligned}
$$

which is the power output of the conventional 1DOF EMEH (Fig. 1b).

\section{Experimental validation}

\subsection{Experimental setup}

Fig. 3 shows the prototype of the proposed 2DOF hybrid P-EMEH. It can be seen that the PEH part is a clamped-clamped piezoelectric beam with a central mass and two piezoelectric elements bonded symmetrically on the brass beam. The two piezoelectric elements are electrically connected in parallel. The EMEH part consists of a spring, a magnet, and a wound coil. One end of the spring is connected with the central mass of the PEH part and the other with the magnet. A copper wound coil is placed on the base, the axis of which aligns with the direction of the motion of the magnet. The material and geometric parameters of the $\mathrm{PEH}$, magnet, and coil are given in Tables 1 and 2, respectively.

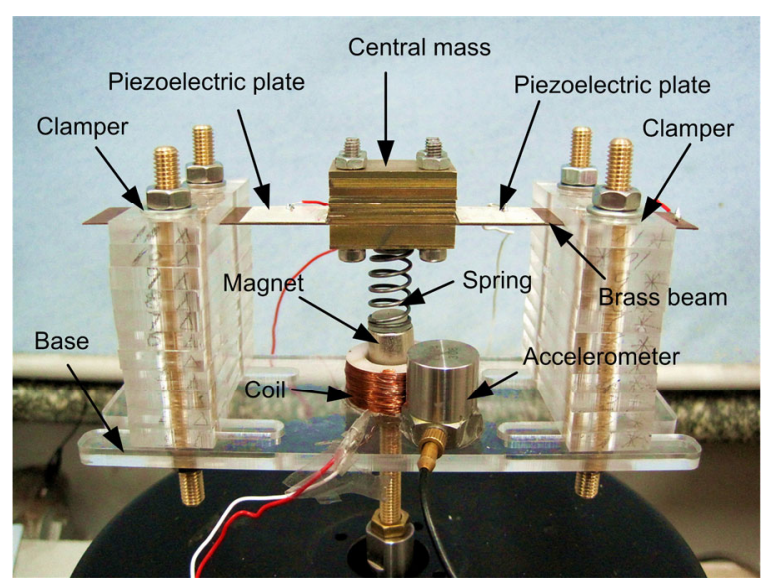

Fig. 3 Experimental prototype of 2DOF hybrid P-EMEH

Table 1 Parameters of PEH part

\begin{tabular}{lc}
\hline \multicolumn{1}{c}{ Parameter } & Value \\
\hline Clamped length of the brass beam $(\mathrm{mm})$ & 80 \\
Width of the brass beam $(\mathrm{mm})$ & 20 \\
Thickness of the brass beam $(\mathrm{mm})$ & 0.5 \\
Thickness of the piezoelectric element $(\mathrm{mm})$ & 0.2 \\
Static clamped capacitance, $C_{\mathrm{p}}(\mathrm{nF})$ & 42 \\
Effective mass of the beam with central mass, & 104 \\
$M_{1}$ (g) & \\
Damping ratio of PEH, $\zeta_{1}$ & 0.014 \\
\hline
\end{tabular}

Table 2 Parameters of magnet and coil

\begin{tabular}{lc}
\hline \multicolumn{1}{c}{ Parameter } & Value \\
\hline Effective mass of the spring and the magnet, & 10 \\
$\quad M_{2}(\mathrm{~g})$ & \\
Mass of the coil part $(\mathrm{g})$ & 16 \\
Radius of the magnet, $r_{\mathrm{m}}(\mathrm{mm})$ & 6 \\
Height of the magnet, $h_{\mathrm{m}}(\mathrm{mm})$ & 10 \\
Residual the flux density, $B_{\mathrm{r}}(\mathrm{T})$ & 1.1 \\
Nearest distance between the coil and & 2.8 \\
$\quad$ Magnet, $z_{1}(\mathrm{~mm})$ & 15 \\
Height of the wounded coil $(\mathrm{mm})$ & 52.15 \\
Total length of the coil, $l(\mathrm{~m})$ & 40.6 \\
Inductance of the coil, $L_{\text {coil }}(\mathrm{mH})$ & 116 \\
Resistance of the coil, $R_{\text {coil }}(\Omega)$ & 0.0035 \\
Damping ratio of EMEH, $\zeta_{2}$ & \\
\hline
\end{tabular}


It should be mentioned that if the coil is opencircuited, there is no power output from the EMEH part and the electromagnetic backward coupling force disappears. Thus, the system behaves as a $2 \mathrm{DOF}$ $\mathrm{PEH}$. If the piezoelectric elements are short-circuited, there is no power output from the PEH part and the piezoelectric backward coupling force disappears. Thus, the energy harvesting system behaves as a 2DOF EMEH. If the spring is detached from the central mass, the system degrades to a $1 \mathrm{DOF}$ PEH. If we replace the current brass beam with a much thicker and thus much stiffer one, the relative displacement between the central mass and the base can be neglected and the EMEH part can be regarded as a 1DOF EMEH subjected to the base excitation. We will compare the performances of these five configurations in the experiment and validate the derived theoretical models later.

Fig. 4 shows the experimental setup. The prototype 2DOF hybrid P-EMEH is mounted on an electrodynamic shaker. An accelerometer is mounted on the platform of the harvester to measure the base excitations. The shaker is connected to a signal generator through a power amplifier. The signal generator and power amplifier are tuned manually to provide the desired excitation to the system. The output terminals of the piezoelectric elements and the wound coil are connected to two respective variable resistors. The power outputs from the PEH part and the EMEH part are added at each frequency and we searched for the optimal total power by altering the two resistors.

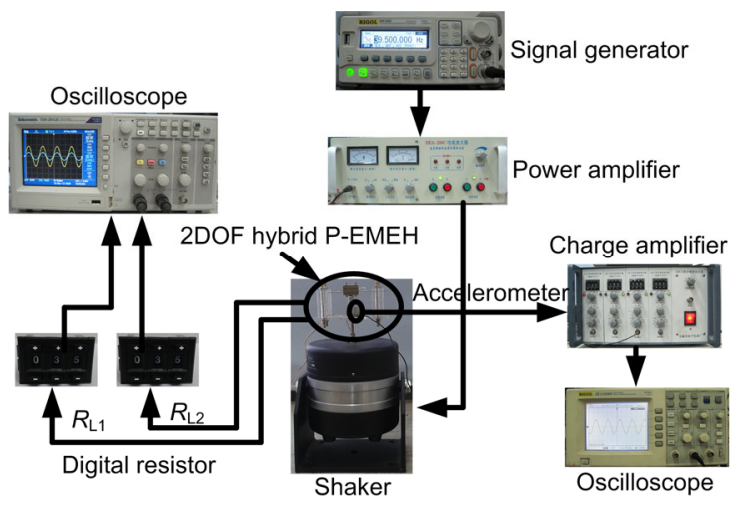

Fig. 4 Experiment setup of 2DOF hybrid P-EMEH

Prior to the model validation, the system parameters should be determined. The average magnetic flux density of the cylindrical magnet, $B_{1}$, is ap- proximated by considering the coil divided into $n$ sections,

$$
B_{1}=\frac{B_{\mathrm{r}}}{2} \frac{1}{n} \sum_{i=1}^{n}\left[\frac{z_{i}+h_{\mathrm{m}}}{\sqrt{\left(z_{i}+h_{\mathrm{m}}\right)^{2}+r_{\mathrm{m}}^{2}}}-\frac{z_{i}}{\sqrt{z_{i}^{2}+r_{\mathrm{m}}^{2}}}\right],
$$

where $z_{i}$ is the distance from the magnet to the $i$ th cross section of the coil. Between the nearest distance from the magnet $z_{1}=2.8 \mathrm{~mm}$ and the farthest distance $z_{n}=17.8 \mathrm{~mm}$, we consider increments of $0.1 \mathrm{~mm}$ and thus $n=151$. According to Eq. (17), the average magnetic flux density $B_{1}=0.0789 \mathrm{~T}$.

Other parameters can be determined from the experiment. Due to the piezoelectric coupling, 1DOF PEH (removing the spring and magnet) possesses a short circuit resonant frequency $f_{\mathrm{sc}}$ (when the load resistance approaches 0 ) and an open circuit resonant frequency $f_{\text {oc }}$ (when the load resistance approaches infinity). In this study, we use two different resistors $\left(R_{\mathrm{L} 1}=100 \Omega\right.$ and $R_{\mathrm{L} 1}=1 \mathrm{M} \Omega$ ) to obtain the voltage frequency responses of $1 \mathrm{DOF} \mathrm{PEH}$, which approximate the short circuit and open circuit conditions, respectively. According to these responses, $f_{\mathrm{sc}}$ and $f_{\mathrm{oc}}$ are measured as $81.5 \mathrm{~Hz}$ and $81.8 \mathrm{~Hz}$, respectively. The EMCC of the 1DOF PEH can be calculated as 0.086 according to the equation $k_{\mathrm{p}}=$ $\left[\left(\omega_{\mathrm{oc}} / \omega_{\mathrm{sc}}\right)^{2}-1\right]^{1 / 2}$, where $\omega_{\mathrm{sc}}$ and $\omega_{\mathrm{oc}}$ are the short circuit and open circuit resonant angular frequencies, respectively. $\omega_{\mathrm{sc}}=2 \pi f_{\mathrm{sc}}$ and $\omega_{\mathrm{oc}}=2 \pi f_{\mathrm{oc}}$. Note that in Eq. (7), $\omega_{1}$ refers to the natural angular frequency of the mechanical structure for the 1DOF PEH without considering the backward piezoelectric coupling effect, i.e., the piezoelectric element approximates the short circuit. Thus, $\omega_{1}=\omega_{\mathrm{sc}}=512.08 \mathrm{rad} / \mathrm{s}$. Similarly, $\omega_{2}$ refers to the natural angular frequency of the mechanical structure for 1DOF EMEH (the spring with the magnetic element only) without considering the backward electromagnetic coupling effect, i.e., the coil is open circuit. According to the voltage frequency response, the open circuit resonance frequency $f_{2}$ is measured as $76 \mathrm{~Hz}$, and thus, $\omega_{2}=2 \pi f_{2}=477.52 \mathrm{rad} / \mathrm{s}$. Furthermore, the frequency ratio $\alpha=\omega_{2} / \omega_{1}=0.9325$. The EMCC of the $1 \mathrm{DOF}$ EMEH can be calculated as 0.4277 according to the equation $k_{\mathrm{e}}=\theta_{\mathrm{e}} /\left(L_{\text {coil }} K_{2}\right)^{1 / 2}$, where $K_{2}=M_{2} \omega_{2}^{2}$. The damping ratios $\zeta_{1}$ and $\zeta_{2}$ of the 1DOF PEH and 1DOF EMEH, which are also the damping ratios of the PEH 
part and EMEH part in the 2DOF hybrid P-EMEH, can be determined by the log decrement method from the voltage attenuation curves of the PEH and EMEH, respectively.

$$
\zeta=\frac{1}{2 \pi k} \ln \left(\frac{A_{1}}{A_{k+1}}\right),
$$

where $A_{1}$ is the first amplitude of the attenuation curve, and $k$ is the decaying cycle. The damping ratios of $\zeta_{1}$ and $\zeta_{2}$ are given in Tables 1 and 2, respectively.

\subsection{Results}

Figs. $5 \mathrm{a}$ and $5 \mathrm{~b}$ show the experimental and simulation results from various harvester configurations, respectively. The experiment and simulation are both conducted under an excitation level of $1 \mathrm{~m} / \mathrm{s}^{2}$ (root mean square value). Note that the 1DOF PEH has a sole peak of $0.69 \mathrm{~mW}$ and the 1DOF EMEH has a sole peak of $0.48 \mathrm{~mW}$ in the frequency range of $65 \mathrm{~Hz}-$ $95 \mathrm{~Hz}$. The 2DOF energy harvester prototypes (2DOF PEH, 2DOF EMEH, and the proposed 2DOF hybrid P-EMEH) can achieve two peaks in this frequency range, providing wider operating bandwidth. To further compare the maximum power outputs of the five harvester configurations, they are divided into two groups. One group includes 1DOF PEH, 2DOF PEH, and 2DOF hybrid P-EMEH, and the other group includes 1DOF EMEH, 2DOF EMEH, and 2DOF hybrid P-EMEH. Note that in Fig. 5a, the 2DOF hybrid P-EMEH has improved the maximum power output $(2.16 \mathrm{~mW})$ as compared to the 2DOF PEH $(0.96 \mathrm{~mW})$ and 1DOF PEH $(0.69 \mathrm{~mW})$. Similarly, the 2DOF hybrid P-EMEH also provides a better performance than the 2DOF EMEH $(1.68 \mathrm{~mW})$ and 1DOF EMEH $(0.48 \mathrm{~mW})$. In addition, note that the second mode of the 2DOF hybrid P-EMEH provides comparable output as 1DOF PEH and 1DOF EMEH. Furthermore, it is worth mentioning that the 2DOF hybrid P-EMEH has improved the maximum power density $(16.6 \mathrm{~mW} / \mathrm{kg})$ as compared to the other 2DOF configurations (2DOF PEH, $8.4 \mathrm{~mW} / \mathrm{kg}$; 2DOF EMEH, $12.9 \mathrm{~mW} / \mathrm{kg}$ ) and the 1DOF PEH $(6.6 \mathrm{~mW} / \mathrm{kg})$. Though it is slightly lower than that of the 1DOF EMEH $(18.5 \mathrm{~mW} / \mathrm{kg})$, the much enhanced maximal power output $(2.16 \mathrm{~mW})$ achieved and two close resonant modes (for wider bandwidth) of the 2DOF hybrid P-EMEH as compared to the 1DOF
EMEH are worth this slight sacrifice in power density.

Fig. $5 \mathrm{~b}$ shows the analytical solution with the system parameters determined from experiment (Tables 1 and 2). Note that in Fig. 5b, the predicted maximum power outputs for various harvester configurations and the overall trends of the analytical prediction are quite consistent with the experiment results (Fig. 5a).
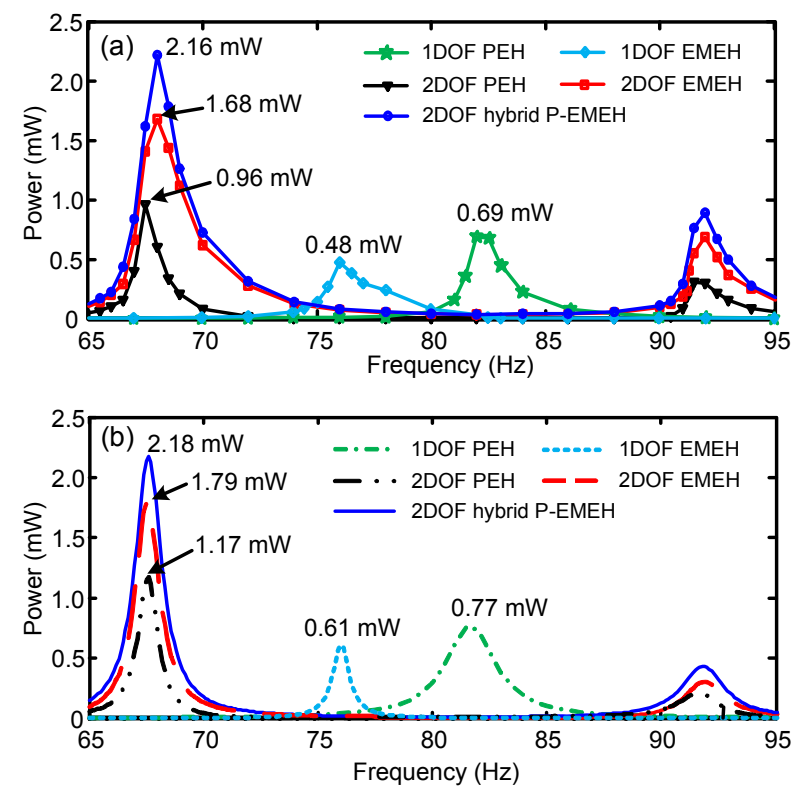

Fig. 5 Power outputs from various configurations for $k_{\mathrm{p}}=0.086$ and $\boldsymbol{k}_{\mathrm{e}}=0.4277$ : (a) experiment and (b) simulation

Both experimental and simulation results indicate that with current system parameters (e.g., coupling coefficients), the hybrid scheme can provide advantageous performance over the stand-alone piezoelectric or electromagnetic techniques.

\section{Effects of electromechanical coupling}

With the validated 2DOF hybrid P-EMEH model, we further study the effects of the EMCC on the maximum power outputs. First, we investigate how the EMCC affects the overall performance and how the two conversion mechanisms affect each other in a 2DOF hybrid P-EMEH model. Subsequently, we compare the effect of the EMCC on power outputs of various harvester configurations including $1 \mathrm{DOF}$ 
PEH, 2DOF PEH, 1DOF EMEH, 2DOF EMEH, and 2DOF hybrid P-EMEH models.

Figs. $6 \mathrm{a}$ and $6 \mathrm{~b}$ show the maximum power outputs from piezoelectric and electromagnetic transducers used in a 2DOF hybrid P-EMEH against two effective EMCCs $k_{\mathrm{p}}$ and $k_{\mathrm{e}}$, respectively. In Fig. 6a, the electromagnetic coupling $k_{\mathrm{e}}$ is fixed $\left(k_{\mathrm{e}}=0.4277\right)$ and the piezoelectric coupling $k_{\mathrm{p}}$ increases. Before the saturation of power output, an increase of $k_{\mathrm{p}}$ leads to an increase in piezoelectric and total power outputs, and a decrease in electromagnetic power output. Similarly, in Fig. $6 \mathrm{~b}, k_{\mathrm{p}}$ is fixed $\left(k_{\mathrm{p}}=0.086\right)$ and the electromagnetic coupling $k_{\mathrm{e}}$ increases. Before the saturation of power output, an increase of $k_{\mathrm{e}}$ leads to an increase in electromagnetic and total power outputs, and a decrease in piezoelectric power output. These results indicate that when piezoelectric and electromagnetic transducers exist in an energy harvesting device, they interact in an opposite way, i.e., the increase of the power output from one electromechanical transducer by increasing its coupling will lead to the decrease of the power output from the other. The main reason is that for a $2 \mathrm{DOF}$ hybrid P-EMEH system, both piezoelectric and electromagnetic coupling will induce electrical damping and suppress the vibrations of the system. In Fig. 6a, as $k_{\mathrm{p}}$ increases, more energy is converted into electricity via the $\mathrm{PEH}$ part and so induces more damping and thus suppresses the vibrations of the system. Since $k_{\mathrm{e}}$ is fixed, the suppressed vibrations result in less contribution of power output from EMEH part. Similarly, in Fig. 6b, as $k_{\mathrm{e}}$ increases, more energy is harnessed via the EMEH part and so induces more damping to the system. Since $k_{\mathrm{p}}$ is fixed, the suppressed vibrations result in the decrease of power output from the PEH part.

Figs. $7 \mathrm{a}$ and $7 \mathrm{~b}$ show the maximum power outputs from various harvester configurations against two effective EMCCs $k_{\mathrm{p}}$ and $k_{\mathrm{e}}$, respectively. It can be seen from Fig. $7 \mathrm{a}$ that an increase of $k_{\mathrm{p}}$ leads to an increase in power outputs in the 1DOF PEH, 2DOF $\mathrm{PEH}$, and 2DOF hybrid P-EMEH models first, but finally the power outputs reach saturation in each case. The trends of power versus coupling from these energy harvesters are the same as the 1DOF PEH in previous studies (Guyomar et al., 2005; Liao and Sodano, 2008). Similarly, in Fig. 7b, an increase of $k_{\mathrm{e}}$
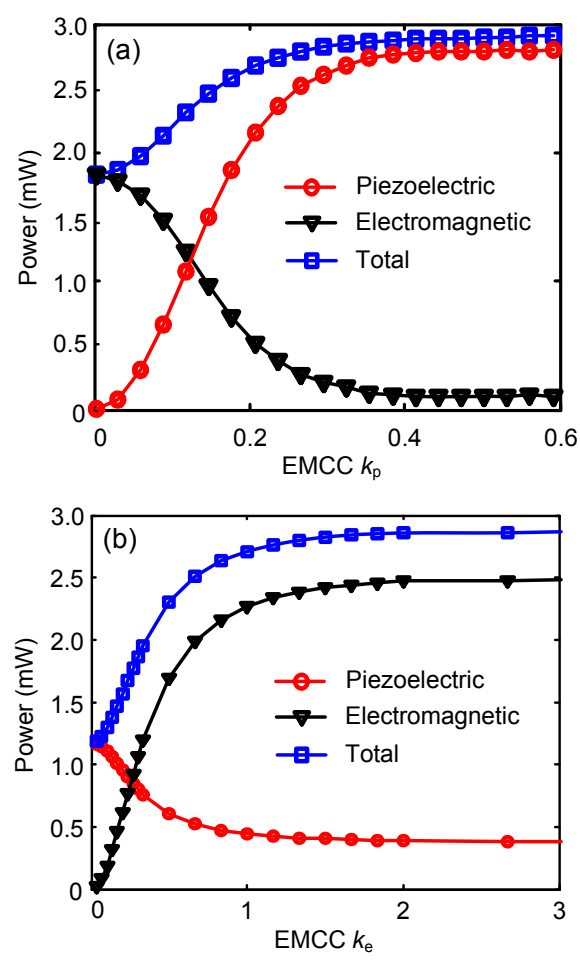

Fig. 6 Maximum power outputs from 2DOF hybrid P-EMEH versus piezoelectric EMCC $k_{\mathrm{p}}\left(k_{\mathrm{e}}=0.4277\right)(\mathrm{a})$ and electromagnetic EMCC $\boldsymbol{k}_{\mathrm{e}}\left(\boldsymbol{k}_{\mathrm{p}}=\mathbf{0 . 0 8 6}\right)(\mathrm{b})$
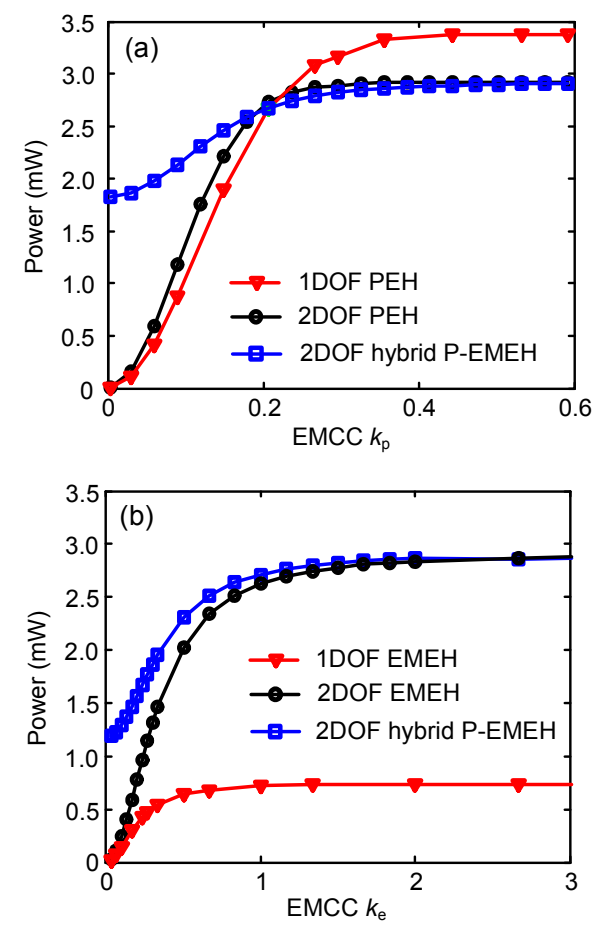

Fig. 7 Maximum power outputs from various harvester configurations versus piezoelectric EMCC $k_{\mathrm{p}}\left(k_{\mathrm{e}}=\mathbf{0 . 4 2 7 7}\right)$ (a) and electromagnetic EMCC $k_{\mathrm{e}}\left(\boldsymbol{k}_{\mathrm{p}}=\mathbf{0 . 0 8 6}\right)(\mathrm{b})$ 
leads to an increase in power outputs from the $1 \mathrm{DOF}$ EMEH, 2DOF EMEH, and 2DOF hybrid P-EMEH models first, but finally the power outputs reach saturation in each case. The trends of power versus coupling are similar to the 1DOF EMEH in previous study (Challa et al., 2013). Moreover, both 2DOF EMEH and 2DOF hybrid P-EMEH have significantly improved power outputs as compared to the $1 \mathrm{DOF}$ EMEH.

To investigate the performance of the energy harvesters in different coupling regions, some researchers (Shu and Lien, 2006) proposed using the parameter $\gamma_{1}=k_{\mathrm{p}}{ }^{2} / \zeta_{1}$ as an indicator of the piezoelectric coupling strength of $1 \mathrm{DOF}$ PEH and proposed the criterion of strong coupling, i.e., $k_{\mathrm{p}}^{2} / \zeta_{1} \geq 4\left(\zeta_{1}+1\right)$ for an $\mathrm{AC}$ interface circuit (pure resistor). Applying this criterion and considering $\zeta_{1}=0.014$ in this work, we have $k_{\mathrm{p}} \geq 0.238$ for the strong coupling of 1DOF PEH. For $k_{\mathrm{p}}=0.238$, the power output corresponds to $90 \%$ of the saturation power output. Similarly, some researchers (Challa et al., 2013) proposed using the parameter $\gamma_{2}=\theta_{\mathrm{e}}^{2} /\left(R_{\text {coil }} C_{2}\right)$ to indicate the coupling strength of the 1DOF EMEH and studied the power outputs when loosely coupled $\left(\gamma_{2} \ll 1\right)$, critically coupled $\left(\gamma_{2}=1\right)$, and strongly coupled $\left(\gamma_{2} \gg 1\right)$. The equation can also be written as $\gamma_{2}=L_{\text {coil }} \omega_{2} k_{\mathrm{e}}{ }^{2} /\left(2 R_{\text {coil }} \zeta_{2}\right)$, where $k_{\mathrm{e}}{ }^{2}=\theta_{\mathrm{e}}{ }^{2} /\left(L_{\text {coil }} K_{2}\right), \quad \zeta_{2}=C_{2} /\left(2 M_{2} \omega_{2}\right)$, and $\omega_{2}=$ $\left(K_{2} / M_{2}\right)^{1 / 2}$. Challa et al. (2013) did not clearly provide the criterion for quantitatively defining the boundaries of coupling strength. We apply a similar criterion as $1 \mathrm{DOF} \mathrm{PEH}$, i.e., $90 \%$ of saturation power output, to define the strong coupling of 1DOF EMEH. We obtained the critical value of $k_{\mathrm{e}}=0.66$ with the system parameters in our work. Considering $L_{\text {coil }}=40.6 \mathrm{mH}$, $R_{\text {coil }}=116 \Omega, \zeta_{2}=0.0035$, and $\omega_{2}=477.52 \mathrm{rad} / \mathrm{s}$ in our work, $\gamma_{2}=10.4$. The result is consistent with the condition of a strongly coupled system $\left(\gamma_{2} \gg 1\right)$ in (Challa et al., 2013). This implies that $90 \%$ of saturation power output can be used to define the strong coupling of 1DOF PEH and 1DOF EMEH. In addition, to our best knowledge, there is no literature discussing the effect of coupling on the performance of a $2 \mathrm{DOF}$ harvester system, no matter whether it is purely piezoelectric, electromagnetic, or hybrid. We apply $90 \%$ of saturation power output to define strong coupling for 2DOF hybrid P-EMEH. For the 2DOF hybrid system, due to the opposite interaction between the two electromechanical couplings, we can only determine the critical value of $k_{\mathrm{p}}$ for strong coupling when $k_{\mathrm{e}}$ is given, and vice versa. In Fig. $7 \mathrm{a}$, given $k_{\mathrm{e}}=0.4277,90 \%$ of saturation power output requires $k_{\mathrm{p}}=0.2$. Hence, $0<k_{\mathrm{p}}<0.2$ is regarded as the weak and medium coupling range. Given $k_{\mathrm{p}}$ in the range of $0-0.2$, the 2DOF hybrid P-EMEH has an improved power output as compared to the 1DOF PEH and 2DOF PEH. Beyond the critical value of $k_{\mathrm{p}}=0.2$, both 2DOF PEH and 2DOF hybrid P-EMEH are near the saturation of power, and the power output of the 2DOF hybrid P-EMEH has no advantage over the 2DOF PEH and is even smaller than that of the 1DOF PEH. Similarly, in Fig. $7 \mathrm{~b}$, given $k_{\mathrm{p}}=0.086,90 \%$ of saturation power output requires $k_{\mathrm{e}}=0.9$. Hence, $0<k_{\mathrm{e}}<0.9$ is regarded as the weak and medium coupling range. Given $k_{\mathrm{e}}$ in the range of $0-0.9$, the 2DOF hybrid P-EMEH provides better performance than the 2DOF EMEH and 1DOF EMEH. These results suggest that the hybrid energy harvester configuration is advantageous over the stand-alone harvester configurations in both weak and medium coupling regimes.

\section{Conclusions}

This paper presents a 2DOF hybrid P-EMEH design combing piezoelectric and electromagnetic transduction mechanisms. The mathematical model of the 2DOF hybrid P-EMEH is established and validated experimentally. The prototype 2DOF hybrid $\mathrm{P}$-EMEH demonstrates its advantageous performance in terms of both wider bandwidth and improved power magnitude as compared to the conventional $1 \mathrm{DOF}$ and $2 \mathrm{DOF}$ harvester configurations with stand-alone conversion mechanisms. With the validated mathematical model, the effect of the piezoelectric and electromagnetic coupling coefficients on the performances of various harvester configurations is analyzed. For the 2DOF hybrid P-EMEH model, although the increase of the power output from one electromechanical transducer will lead to the decrease of the power output from the other, the total power output of the 2DOF hybrid P-EMEH model increases before it reaches its saturation. In the weak and medium coupling scenarios, the hybrid 2DOF energy harvester configuration provides an efficient way to enhance device performance. 


\section{References}

Aldraihem, O., Baz, A., 2011. Energy harvester with dynamic magnifier. Journal of Intelligent Material Systems and Structures, 22(6):521-530. [doi:10.1177/1045389X1140 2706]

Arafa, M., Akl, W., Aladwani, A., et al., 2011. Experimental implementation of a cantilevered piezoelectric energy harvester with a dynamic magnifier. Proceedings of SPIE, Active and Passive Smart Structures and Integrated Systems, San Diego, USA, p.79770Q. [doi:10.1117/12. 880689]

Challa, V.R., Prasad, M.G., Fisher, F.T., 2009. Coupled piezoelectric-electromagnetic energy harvesting technique for achieving increased power output through damping matching. Smart Materials and Structures, 18(9):095029. [doi:10.1088/0964-1726/18/9/095029]

Challa, V.R., Cheng, S., Arnold, D.P., 2013. The role of coupling strength in the performance of electrodynamic vibrational energy harvesters. Smart Materials and Structures, 22(2):025005. [doi:10.1088/0964-1726/22/2/ 025005]

Dutoit, N.E., Wardle, B.L., Kim, S.G., 2005. Design considerations for MEMS-scale piezoelectric mechanical vibration energy harvesters. Integrated Ferroelectrics, 71(1):121-160. [doi:10.1080/10584580590964574]

Elvin, N.G., Elvin, A.A., 2011. An experimentally validated electromagnetic energy harvester. Journal of Sound and Vibration, 330(10):2314-2324. [doi:10.1016/j.jsv.2010. 11.024]

Guyomar, D., Badel, A., Lefeuvre, E., et al., 2005. Toward energy harvesting using active materials and conversion improvement by nonlinear processing. IEEE Transctions on Ultrasonics, Ferroelectrics, and Frequency Control, 52(4):584-595. [doi:10.1109/TUFFC.2005.1428041]

Harne, R.L., 2012. Theoretical investigations of energy harvesting efficiency from structural vibrations using piezoelectric and electromagnetic oscillators. The Journal of the Acoustical Society of America, 132(1):162-172. [doi:10.1121/1.4725765]

Jung, H.J., Kim, I.H., Koo, J.H., 2011. A multi-functional cable-damper system for vibration mitigation, tension estimation and energy harvesting. Smart Structures and Systems, 7(5):379-392. [doi:10.12989/sss.2011.7.5.379]

Khaligh, A., Zeng, P., Wu, X., et al., 2008. A hybrid energy scavenging topology for human-powered mobile electronics. Proceedings-34th Annual Conference of the IEEE Industrial Electronics Society, Orlando, USA, p.448-453. [doi:10.1109/IECON.2008.4757995]

Kim, H., Kim, S.M., Son, H., et al., 2012. Enhancement of piezoelectricity via electrostatic effects on a textile platform. Energy \& Environmental Science, 5(10):89328936. [doi:10.1039/c2ee22744d]

Lallart, M., Pruvost, S., Guyomar, D., 2011. Electrostatic energy harvesting enhancement using variable equivalent permittivity. Physics Letters A, 375(45):3921-3924. [doi:10.1016/j.physleta.2011.09.043]
Liao, Y.B., Sodano, H.A., 2008. Model of a single mode energy harvester and properties for optimal power generation. Smart Materials and Structures, 17(6): 065026. [doi:10.1088/0964-1726/17/6/065026]

Lumentut, M.F., Howard, I.M., 2011. Analytical modeling of self-powered electromechanical piezoelectric bimorph beams with multidirectional excitation. International Journal of Smart and Nano Materials, 2(3):134-175. [doi:10.1080/19475411.2011.592868]

Roundy, S., Wright, P.K., Rabaey, J., 2003. A study of low level vibrations as a power source for wireless sensor nodes. Computer Communications, 26(11):1131-1144. [doi:10.1016/S0140-3664(02)00248-7]

Shan, X.B., Guan, S.W., Liu, Z.S., et al., 2013. A new energy harvester using a piezoelectric and suspension electromagnetic mechanism. Journal of Zhejiang UniversitySCIENCE A (Applying Physics \& Engineering), 14(12): 890-897. [doi:10.1631/jzus.A1300210]

Shu, Y.C., Lien, I.C., 2006. Analysis of power output for piezoelectric energy harvesting systems. Smart Materials and Structures, 15(6):1499-1512. [doi:10.1088/09641726/15/6/001]

Tadesse, Y., Zhang, S., Priya, S., 2009. Multimodal energy harvesting system: piezoelectric and electromagnetic. Journal of Intelligent Material Systems and Structures, 20(5):625-632. [doi:10.1177/1045389X08099965]

Tang, L.H., Yang, Y.W., 2012. A multiple-degree-of-freedom piezoelectric energy harvesting model. Journal of Intelligent Material Systems and Structures, 23(14): 1631-1647. [doi:10.1177/1045389X12449920]

Tang, L.H., Yang, Y.W., Soh, C.K., 2013. Broadband vibration energy harvesting techniques. Elvin, N., Erturk, A. (Eds.), Advances in Energy Harvesting Methods. Springer, New York, USA, p.17-61.

Wacharasindhu, T., Kwon, J.W., 2008. A micromachined energy harvester from a keyboard using combined electromagnetic and piezoelectric conversion. Journal of Micromechanics and Microengineering, 18(10):104016. [doi:10.1088/0960-1317/18/10/104016]

Wang, H.Y., Shan, X.B., Xie, T., 2012. An energy harvester combining a piezoelectric cantilever and a single degree of freedom elastic system. Journal of Zhejiang UniversitySCIENCE A (Applying Physics \& Engineering), 13(7): 526-537. [doi:10.1631/jzus.A1100344]

Wang, H.Y., Tang, L.H., Shan, X.B., et al., 2014. Modeling and performance evaluation of a piezoelectric energy harvester with segmented electrodes. Smart Structures and Systems, 14(2):247-266. [doi:10.12989/sss.2014.14. 2.247]

Williams, C.B., Yates, R.B., 1996. Analysis of a micro-electric generator for microsystems. Sensors and Actuators A: Physical, 52(1-3):8-11. [doi:10.1016/0924-4247(96) 80118-X]

Yang, B., Lee, C., Kee, W.L., et al., 2010. Hybrid energy harvester based on piezoelectric and electromagnetic mechanisms. Journal of Micro/Nanolithography, MEMS, 
and MOEMS, 9(2):023002. [doi:10.1117/1.3373516]

Yang, Y.W., Tang, L.H., Li, H.Y., 2009. Vibration energy

harvesting using macro-fiber composite. Smart Materials and Structures, 18(11):115025. [doi:10.1088/0964-1726/ $18 / 11 / 115025]$

\section{中文概要:}

本文题目: 一种两自由度的压电一电磁复合能量收集器

A 2DOF hybrid energy harvester based on combined piezoelectric and electromagnetic conversion mechanisms

研究目的：对一种两自由度的压电一电磁复合能量收集器进行发电性能研究。

创新要点: 建立了一种两自由度压电一电磁复合能量收集器发电性能的数学模型, 该数学模型可以评估 两自由度压电-电磁复合能量收集器中压电元件、电磁元件以及系统总输出功率。

研究方法: 对一种两自由度的压电一电磁复合能量收集器进行数学建模, 并实验验证数学模型的正确性。 基于实验测试得到的系统参数值, 理论研究压电元件和电磁元件的机电耦合系数对不同能量 收集器发电性能的影响关系, 并对几种能量收集器的发电能力进行对比分析。

重要结论: 对于非强耦合 (弱或中间耦合) 的两自由度机电转换器, 复合能量收集器 (压电+电磁) 具 有比单一能量收集器 (压电或电磁) 更高的发电能力。

关键词组: 振动; 复合能量收集; 两自由度模型; 压电; 电磁 